\title{
Affrontare l'emergenza tra paura, trauma, regressione
}

\author{
Leonardo Speri \\ Psicologo, psicoterapeuta, psicosocioanalista, presidente di ARIELE Psicoterapia
}

\section{Parte II: prove di resilienza}

\section{Diverse attitudini, diverse suscettibilità}

Cercare di rispondere alla domanda centrale sul perché gruppi e istituzioni in alcuni casi abbandonino le buone pratiche e in altri no, apre a una riflessione sulla differenza tra regressione e resilienza.

$\mathrm{Ma}$ se regressione e resilienza non sono solo proprietà del singolo, bensì dimensioni che assieme alle emozioni transitano tra individuo, gruppo e istituzione, dobbiamo tenere a mente il presupposto che le istituzioni, e i gruppi che le compongono, vanno considerati come organismi viventi e capaci di emozioni gruppali e quindi istituzionali (Box 4).

Per rimanere all'esempio delle TIN, la spinta progettuale e le resistenze rispetto all'apertura ai genitori rappresentano poli opposti in tensione tra loro. E molto frequente che la tensione interna ai singoli e al gruppo, le "emozioni istituzionali", anziché trovare sbocco nell'assunzione della fatica emotiva del cambiamento, induca la ricerca di "aggiustamenti" che non hanno più a che fare con l'applicazione delle evidenze e altri fattori razionali, ma rappresentano il tentativo di placare la tensione stessa: il gruppo fa "come se" si dedicasse al lavoro prefissato, mentre, in realtà, si dedica ad attività di salvaguardia rispetto alle sofferenze e alle diverse emozioni istituzionali, come l'ambivalenza o i vissuti di colpa (Box 5).

Per togliere di mezzo le ansie, i vari professionisti dell'équipe abbandonano una modalità relazionale articolata e complessa, come è quella tra persone, e riducono semplificatoriamente le interazioni a quelle tra ruolo e ruolo, come si osserva quando alla ricerca di una soluzione comune si contrappongono, tipicamente, quei "non tocca a me" o viceversa "è una mia esclusiva” che spesso paralizzano l'evoluzione dei servizi. Ma quando la scissione tra agire, pensare e sentire è massima, la conseguenza è la de-umanizzazione delle relazioni. È una configurazione relazionale interna all'équipe in cui sono anche stati rilevati esiti evidenti sugli outcome di salute, come messo in luce anche da una recente ricerca di Fanelli e altri [2].

\section{Cosa fa la differenza}

Propongo che, al netto di tutte le giustificazioni che vengono in genere addotte, le ragioni delle differenti inclinazioni verso l'inclusione dei genitori da parte dei vari gruppi di lavoro, siano da ricercare nella loro coesione interna, nel loro orientamento al compito, inteso come scopo ultimo, e nella loro conseguente peculiare capacità di risposta emotiva alle sfide poste.

Questo dipende molto anche dagli stili di leadership, che orchestrano e influenzano il "tono" dei rapporti tra tutti gli attori, dalla qualità del percorso formativo e soprattutto del processo trasformativo che ha condotto un'équipe all'adesione a nuovi e più adeguati standard di gestione. I nuovi standard assimilati, che sappiamo più appropriati e coerenti in termini di efficienza ed efficacia, nel momento in cui vogliono garantire un ruolo attivo al genitore nella care, implicano azioni molto più coinvolgenti sul piano personale ed emotivo, come sono l'ascolto autentico della persona, che sta alla base del counselling, dell'approccio della medicina narrativa, di un approccio alle cure personalizzato e "più umano" (sulla cosiddetta umanizzazione mi soffermerò più avanti).

È completamente diverso se siamo davanti a un'adesione posticcia e "formale" a nuovi "standard" piuttosto che a un ripensamento, magari travagliato, delle pratiche tradizionali e delle modalità relazionali più croniche, che ha permesso l'interiorizzazione del senso di una procedura, di un fare - o all'occorrenza anche di un non-fare - più difficile. In altre parole si tratta di quanto si è stati in grado di incardinare il sapere e il saper fare su un saper essere, capace di rendere le competenze embodied, "incorporate", come non solo oggi si raccomanda.

\section{Resilienza vs regressione}

A parità delle difficoltà che comporta l'accesso dei genitori, per esempio, è completamente diverso il significato del messaggio "mi dispiace signora, non possiamo più farvi entrare, ma stia tranquilla, ci pensiamo noi" dal cercare di garantire, nei fatti, una continuità di presenza anche nelle situazioni meno favorevoli, guadagnare ogni spazio possibile perché "il suo piccolo e anche noi abbiamo bisogno di lei". Penso che tutti ci rendiamo conto della fondamentale diversità tra le due risposte, raccolte in due TIN diverse.

La prima risposta può essere considerata regressiva, perché intesa proprio come il ritorno a uno stadio precedente dello sviluppo organizzativo, a una fase sentita più sicura, a una configurazione più protettiva relativamente al contagio per il piccolo e per se stessi, rispetto anche a un altro contagio, non solo virale, ma, come vedremo, psichico ed emotivo.

La seconda risposta ha a che fare con la ricerca, faticosa, di soluzioni capaci di contemperare l'irrinunciabile apporto di salute rappresentato dalla presenza dei genitori e l'altrettanto ineludibile necessità di protezione dei propri pazienti e di se stessi, fondamentale in ogni emergenza e non solo, perché quest'ultima non sopprima la tensione verso l'azione più appropriata, cioè l'apertura ai genitori. Questa flessibilità è caratteristica della resilienza, non della regressione.

\section{Complessità vs semplificazione}

La prima risposta è nell'ordine della semplificazione; triste semplificazione, perché con l'esclusione dei genitori ignora e sperpera un patrimonio di risorse irrinunciabile, oltre a renderli e farli sentire inutili (quello che definiamo dis-empowerment). La frase "le tolgo il pensiero", finisce per avere in questi casi una valenza tutt'altro che metaforica, in particolare dopo che il pensiero, come abbiamo visto, è già stato attaccato duramente dall'esperienza traumatica.

La seconda è più difficile, perché è nell'ordine della complessità, della consapevolezza e della valutazione dei rischi e dei benefici della presenza e, nel momento in cui viene a essere in qualche modo ridimensionato l'ingresso, è capace di attivare un accudimento del patrimonio affettivo/ terapeutico rappresentato dai genitori.

In questo secondo caso l'incontro frequente degli operatori con i genitori, singolarmente, in gruppo (risorsa enorme), la spremitura del latte, e ogni altra forma di coinvolgimento, forniscono un aiuto concreto 
e restituiscono almeno in parte un ruolo attivo.

\section{Ambivalenze}

Per comprendere meglio come i diversi gruppi di lavoro vivano queste situazioni può venire utile una constatazione "clinica", rispetto al tema della presenza dei genitori: ci sono operatori che in modo più o meno frequente ed esplicito esprimono un sollievo e la sensazione di lavorare meglio in loro assenza.

Questi professionisti sono portavoce di una delle parti della dimensione ambivalente che probabilmente abita in tutti: da un lato c'è la fatica del confronto coi genitori, che in alcuni diventa prevalente, dall'altro c'è invece il sincero desiderio di averli alleati, che non esclude la scomodità del confronto, ma trae forza dalla consapevolezza che i genitori rappresentano un'opportunità fondamentale ai fini della cura.

Quando una delle due posizioni prende il sopravvento, produce massa critica, pervade l'intero gruppo di lavoro e ne determina le scelte, in una direzione o nell'altra. Oppure, come ogni tanto possiamo vedere, viene messa in scena la dialettica in corso, con la differenza di comportamento dei diversi turni di servizio o da singoli operatori.

Questa oscillazione, che è dentro a ciascuno di noi come singoli, nella nostra controversia interiore, si materializza nel gruppo di lavoro, e a ben vedere anche a un livello più alto.

Pensiamo a un esempio di come in questa dialettica sia prevalsa la voce inclusiva: la costruzione della alleanza tra società scientifica e organizzazioni dei genitori. Nel segno di un riconoscimento reciproco è stato avviato da tempo un percorso di collaborazione istituzionale tra neonatologi e genitori (tra SIN e la rete delle associazioni riunite in Vivere Onlus) [3], su obiettivi comuni come le raccomandazioni per "La promozione dell'allattamento materno nelle TIN e accesso dei genitori in reparto" o la redazione del "Manifesto dei diritti del bambino nato prematuro", riuscendo a ottenere ascolto ed essere incisivi a livello ministeriale e oggi uniti a tanti altri alleati nella campagna "Zero Separation" [4]. Una collaborazione analoga è auspicabile anche a livello periferico, nelle singole TIN, dove i genitori "senior" con le loro associazioni, dovrebbe esseri inclusi nella discussione su come attuare al meglio le raccomandazioni e applicare localmente le buone pratiche.

\section{Lontano dagli occhi.}

Tuttavia credo sia altrettanto importante e utile non eludere l'altra polarità, e stu-

\section{BoX 4. Emozioni e benessere istituzionale}

È uno degli obiettivi della psicosocioanalisi studiare come le emozioni individuali riverberino e si trasformino nel singolo, nella coppia, nella famiglia, nei gruppi, nelle istituzioni, nelle comunità più o meno estese (la polis). L'individuo è la particella elementare, la cellula; il gruppo di lavoro è un organo che assieme ad altri compone il corpo istituzionale: il suo benessere e quello del suo contesto istituzionale si influenzano reciprocamente. Coerentemente con l'epistemologia della complessità: "Un'istituzione non è un oggetto inanimato ma un sistema vivente dotato di una sua organizzazione. È noto che alcune istituzioni rispetto ad altre godono di una maggiore salute, di un livello di maggior benessere organizzativo con vistosi esiti proprio sulla capacità di perseguire obiettivi sfidanti. Si sa anche che questo esito non è casuale, ma frutto di un adeguato e costante processo trasformativo nel quale c'è ascolto e attenzione, coinvolgimento e investimento sul valore delle emozioni, ossia sulla qualità delle relazioni professionali in essere. Un'istituzione in salute è un soggetto collettivo articolato e complesso che persegue obiettivi comuni, vive il suo tempo, si trasforma, apprende" (esergo del manuale di GenitoriPiù, da vari scritti psicosocioanalitici di Ermete Ronchi).

\section{BOX 5. II lavoro del gruppo, tra obiettivo e sopravvivenza}

L'équipe di una TIN è anche un contenitore dove le indicazioni scientifiche da un lato e le emozioni istituzionali dall'altro si incontrano nella quotidianità del rapporto con i propri assistiti con i quali danno vita a un unico sistema di influenze reciproche: I'"assetto psichico" del gruppo di lavoro ha un ruolo determinante nel configurare le modalità relazionali dei singoli operatori nell'incontro con i genitori e la qualità delle relazioni. Quando è all'opera, un gruppo persegue, insieme al fine per cui è nato, suo compito ultimo, anche l'obiettivo della propria sopravvivenza. In certe condizioni il gruppo può sacrificare alla propria sopravvivenza emotiva il perseguimento "onesto" del compito, mistificando come necessarie azioni che invece sono tese a salvaguardare equilibri interni. Si tratta il più delle volte di meccanismi di difesa inconsci, contro le ansie mobilitate dal compito stesso. Spesso il conflitto tra progettualità e resistenza al cambiamento non viene risolto a favore della crescita ma dà origine a soluzioni spurie, falsate, realizzando quella che Pichon-Rivière chiama "I'impostura del compito": il gruppo fa "come se" si dedicasse allo scopo per cui è costituito, mentre, in realtà, si dedica ai "compiti" - le mansioni - che, pur formalmente ineccepibili, gli permettono di "ingannare il tempo" e soprattutto mettersi al riparo dalle emozioni più spiacevoli [1].

diare le ragioni del sentimento di sollievo nel momento in cui non ci si trova più costretti a confrontarsi con $i$ genitori. E fondamentale prima di tutto uscire da un atteggiamento giudicante se vogliamo comprendere e gestire positivamente le dinamiche che ne stanno alla base: quel sollievo è un'emozione che ha la sua dignità, porta un sapere e va ascoltata. Se la ignoriamo presenterà prima o poi il conto.

Ascoltandola il primo pensiero corre al trauma secondario (trauma vicario). L'empatia con chi è traumatizzato ha un elevato costo emotivo, e chi si occupa di traumatizzati è esposto a un contagio psichico tanto più importante, quanto più si identifica con l'altro. Questo meccanismo è fortemente influenzato dalla nostra struttura biologica, come viene dimostrato dagli studi di neurofisiologia (ricordiamo i neuroni specchio), dalle sovrastrutture culturali che l'hanno più o meno valorizzata o messa sotto controllo, da potenti condizionamenti ambientali, in particolare di gruppo e di massa, su cui, purtroppo, abbiamo abbondanza di esempi negativi sconvolgenti.

\section{De-umanizzazione}

Gli esempi più studiati sono di solito quelli inerenti le guerre, soprattutto le più recenti, all'olocausto, in generale alle situazioni estreme, sia relative alle grandi stra- gi terroristiche o alle catastrofi per mano dell'uomo o semplicemente naturali (con valenze emotive diverse): traumi collettivi in cui i singoli, le famiglie, intere comunità sono state esposte all'orrore. È attraverso un processo di de-umanizzazione della vittima che diventano possibili le tante efferatezze che la storia umana ha dovuto registrare.

Utilizzare esempi così forti e devastanti non significa nel modo più assoluto assimilare i comportamenti umani in ambito medico, e specificatamente nelle TIN, a quegli orrori. Purtroppo la storia ci offre tremendi esperimenti naturali, che sta a noi trasformare in occasioni di apprendimento, cercare di riconoscere quali dinamiche si ripetono anche in contesti molto diversi come qualità e portata.

Non esitiamo a definire disumani alcuni comportamenti, ma - per esempio - a cosa ci riferiamo quando parliamo di "umanizzazione delle cure"? La parola umanizzazione, che non piace a molti, e lo condivido, presuppone una qualche forma di disumanità da cui il mondo della cura è chiamato a riscattarsi. Il fastidio per la parola umanizzazione credo derivi proprio dall'avversione all'idea che le configurazioni relazionali di una certa modalità medica (non ancora del tutto) passata siano disumane: in realtà sono state e rimangono umane pur nel loro essere inadeguate, all'occasione anche 
violente [5] e per noi, oggi, profondamente sbagliate, ma proprio perché umane sono passibili di analisi e di trasformazione. Sostiene Hinshelwood: "È una capacità sorprendente degli esseri umani quella di poter abbandonare la preoccupazione per gli altri come persone ed essere coinvolti in una dinamica collettiva a collaborare in un processo disumanizzante" [6].

\section{Disattivare l'empatia}

L'oggettivazione della persona in ospedale, sottraendole con il nome la storia e riducendola con il numero a una diagnosi, è stata, come credo sia ancora, una maniera organizzata di sottrarsi all'insopportabilità dello sguardo dell'altro sofferente. Ce lo insegnano gli studi pioneristici della scuola inglese, in particolare quello, storico di Isabel Menzies del 1959 che per prima coglie lo scopo latente dell'organizzazione del lavoro infermieristico di un ospedale in difficoltà: la spersonalizzazione del contatto per ridurre lo stress della relazione col paziente [7]. Come abbiamo visto a proposito della situazione traumatica, l'eccesso di esposizione emotiva porta a disconnetterci difensivamente dalla sua fonte, rimuovendo quindi nella relazione con l'altro i presupposti per qualsiasi relazione empatica.

Le difese, va ricordato, sono necessarie, indispensabili per sopravvivere psichicamente. I meccanismi di difesa servono per mantenere l'integrità psichica, garantire la convivenza dei nostri diversi movimenti emotivi e organizzare in modo armonico il nostro mondo interno.

L'oggettivazione è, per esempio, una necessità nell'approccio chirurgico, che permette di sospendere l'influenza delle perturbazioni emotive a favore della massima concentrazione sull'organo e sull'azione. Ma fuori dalla sala operatoria, quando entriamo nella dimensione della care, la relazione e i suoi risvolti affettivi sono ineludibili. Le routine, l'organizzazione oraria, la concentrazione rivolta ai protocolli standardizzati e non alla singolarità delle persone fino alla de-umanizzazione, si ergono come difesa contro le ansie connesse alla relazione.

\section{Lo sguardo del genitore}

Ho raccolto il racconto di come giovani infermiere e infermieri di una TIN vivessero con timore la presenza dei genitori durante l'esecuzione di manovre difficoltose, dolorose, e in qualche caso inevitabilmente segnate dall'insuccesso e dalla necessità di ripeterle. Lo sguardo del genitore posto su di sé era vissuto come giudicante. Chiunque attraversi un apprendimento conosce lo stato d'animo iniziale di inadeguatezza e il timore del giudizio, a cui troppo spes- so è assimilata la valutazione. Credo che in questo caso però, oltre alla paura del giudizio, anzi, nascosta da questa, abbiamo a che fare ancora con l'essere a tu per tu con la sofferenza e con l'intrusione delle emozioni in una procedura ad alto tasso tecnico. Si è già detto come il dolore del bambino pretermine non possa essere pienamente decifrabile a un'osservazione diretta: non così lo sguardo del genitore che di quel dolore diventa il portavoce, amplificato dal proprio dolore e dalla propria condizione d'impotenza.

Al di là di questo esempio, i genitori, in quanto espressione del trauma del bambino, del proprio e oggi anche alle prese con quello del Covid-19, sono diventati davvero scomodi, potenzialmente contagiosi fisicamente, ma di sicuro psichicamente.

Temo, ma in alcuni casi è probabile, che la chiusura imposta dalla pandemia abbia comportato un doppio sollievo: la minimizzazione dei rischi di contagio fisico e la minimizzazione del confronto con i genitori. Più crudamente il Covid-19 potrebbe aver fornito un alibi a chi non era poi così profondamente convinto del senso della presenza dei genitori.

\section{Regole come espressione cristallizzata di stati emotivi}

La difesa da queste forme di contagio impegna il singolo professionista, riverbera nel gruppo di lavoro, si struttura ai livelli superiori del sistema, come le direzioni sanitarie, che sono ingaggiate dalla necessità di dare norme rapide ma uniformanti, più semplificatorie che semplici, coerenti con le indicazioni degli organi centrali. Si mescolano obiettiva necessità e medicina difensiva, il che non sembra lasciare spazio a scelte più complesse, a fare le dovute differenze, volta per volta mirate a salvaguardare aspetti essenziali della cura, alla valutazione della specificità di un ambito come quello delle TIN, o del punto nascita, e ancor di meno alla valutazione caso per caso.

Viene "tolto il pensiero" anche agli operatori. Il carattere prescrittivo delle norme è un potente ansiolitico e permette di depositare "nelle pieghe dell'organizzazione", l'insieme complesso e contraddittorio delle emozioni, risolvere l'ambiguità in cui ci si trova immersi.

Quando questo non succede, si assiste - sul lato opposto - a una rinuncia all'assunzione di responsabilità, talora a una latitanza decisionale, o alla produzione di norme ambigue che lasciano irrisolto il conflitto e riversano nella filiera il carico della responsabilità della risoluzione del problema.

La regola, soprattutto in regime di incertezza e di scarsa informazione, cerca di rispondere anzitutto a queste ansie. Credo che possiamo ora capire meglio le parole "ha prevalso il timore" nelle équipe oppure "un diverso atteggiamento professionale" nelle società scientifiche e leggere il riverbero tra ciò che accade all'operatore in prima fila e quello che succede nel suo gruppo di lavoro e poi, in questo caso, nella sua comunità professionale. Quest'ultima, con la redazione delle linee guida si prende in carico un'importante responsabilità attraverso un'azione colma di significato emotivo.

\section{Inversione di tendenza}

Infatti, se da un lato assistiamo a un'amplificazione dell'ansia di livello in livello, assistiamo anche, per fortuna, a quella che un collega ha chiamato felicemente "la catena di s-montaggio dell'ansia”. Ognuno al suo livello, dall'Istituto Superiore di Sanità alle società scientifiche, da quelle direzioni che si sono messe in ascolto dei responsabili delle strutture e, a cascata, degli operatori in prima linea in ascolto dei genitori, in tanti hanno cercato di mantenere vivo uno spazio di pensiero e di non farsi catturare del tutto nel corto circuito "allarme/sovraeccitazione/paralisi o evacuazione nell'azione" al quale rischia di ridurci ogni esperienza traumatica.

Credo che questo spieghi perché in alcuni gruppi di lavoro la paura del contagio fisico e l'esposizione al contagio psichico siano state interpretate come problemi da superare e non ai quali arrendersi, nell'idea prioritaria di garantire al bambino prematuro la pienezza delle cure. Diversamente queste emozioni istituzionali non vengono elaborate e si sedimentano in regole difensive, attingendo al già pensato e già sperimentato, tornando al passato, intraprendendo un cammino regressivo.

\section{II gruppo restauratore di connessione}

Per evitare la deriva regressiva, le emozioni connesse al trauma devono trovare uno spazio in cui essere elaborate e quindi tornare parlabili. Bisogna riprendersi la parola. Bisogna riprendersi il pensiero che ci è stato tolto dal trauma. Bisogna trovare il sostegno che ci permetta di rendere sopportabili le emozioni e di non uccidere l'empatia. Il gruppo ben-disposto è potenzialmente il luogo elettivo di questa elaborazione e di questo sostegno.

$\grave{E}$ anche il luogo dove le ansie legate alla paura possono essere metabolizzate: tutto diventa più sopportabile se non ci sentiamo soli, lo sanno i professionisti, lo sanno ancor di più i genitori. Mettere al mondo e accudire il neonato in solitudine, dove per esempio non è stata permessa la presenza dei padri, o addirittura essere totalmente separati, è stata e continua a essere una 
delle esperienze più dolorose e sconfortanti di questi lunghi mesi.

Stare in un gruppo significa essere in connessione con gli altri: per sua stessa natura il gruppo si pone come antagonista della disconnessione sociale e della rottura dei legami provocata dal trauma. Stare insieme in un gruppo di lavoro esposto ai traumi e alla sofferenza più acuta o logorante è anche l'unico modo per poterle dare un senso e non venirne sopraffatti.

\section{Quando si rompono i legami}

La rottura dell'insieme dei legami tra individui, la cosiddetta "matrice gruppale", è uno degli elementi forti che caratterizzano le catastrofi, le guerre, e altri traumi collettivi.

Nel 1972 gli Stati Uniti sono stati molto scossi da un episodio, il disastro di Buffalo Creek in Virginia [8], un'inondazione di fango e detriti delle miniere, molto simile alla tragedia di Stava del 1985, o a quella del Vajont. I morti furono 125, lontani dagli oltre 2000 del Vajont e dai 268 di Stava. Le comunità colpite però furono ben 17 , con 1100 feriti e 4000 senza tetto: la sindrome di Buffalo Creek fu studiata a lungo anche sui bambini. Lo riporto perché fu oggetto di numerosi studi anche di follow up e per dei ricordi personali legati alla scoperta dell'esistenza di una soggettività collettiva. C'è un'interessante conclusione di uno studio sociologico di alcuni anni dopo che mise in luce nella comunità "tensioni tra individualismo e dipendenza, autoaffermazione e rassegnazione, egocentrismo e orientamento di gruppo, la perdita di connessione, il disorientamento, declino della moralità, aumento della criminalità" [9]. Alla fine si scoprì "che l'inondazione aveva distrutto gli edifici e aveva devastato i legami comuni che tenevano uniti i loro abitanti”. L'incapacità di queste comunità, a differenza di altre, di risollevarsi aveva le sue radici molto prima: le strutture culturali e dei legami sociali proprie delle comunità montane erano state abbandonate con l'arrivo dell'industria mineraria, e non erano state sostituite. Il crollo della diga spazzò via ciò che ne era rimasto. Una comunità, un gruppo sottoposto a uno stress può avere una diversa capacità di sopravvivenza in ragione di come era stato costruito in termini di legame sociale, cioè produzione di nessi sociali, senso di scambio e, nella polis, di cittadinanza e contrattualità, ingredienti del cosiddetto “capitale sociale" [10].

Comunità, istituzioni e gruppi alla prova del Covid-19

Gli interrogativi su quali siano gli effetti della pandemia da Covid-19 sui singo- li e le famiglie, sui gruppi di lavoro e, a cascata, sulla nostra comunità, non troveranno risposte immediate se non tra qualche tempo.

Al di là della prima reazione comunitaria apparentemente coesa, con lo slogan "andrà tutto bene" (che ha rappresentato una reazione - molto umana - di anestetizzare in qualche modo l'ansia con una certa dose di negazione), abbiamo da subito assistito a circoscritti episodi di intolleranza, anche nei confronti dei sanitari, che ci hanno ricordato che le situazioni estreme non spingono solo alla solidarietà ma possono creare anche uno sconvolgimento del patto sociale e mettere a dura prova la tenuta istituzionale, come abbiamo visto di più nella seconda ondata.

Il carattere di queste reazioni collettive variamente distribuite è evidentemente di tipo regressivo e semplificatorio, di divisione tra amici e nemici, tra immuni e contagiosi, tra pro e contro (la mascherina, le misure, ecc.). Scissioni che sono una delle risposte osservate per effetto dei traumi prolungati, tese alla strutturazione di confini illusoriamente protettivi della propria individualità e della propria fragile identità, anche attraverso la negazione o la sottovalutazione del pericolo pandemico.

Ora, il gruppo di lavoro vive comunque in questo contesto sociale e ne è influenzato: così c'è il rischio che per una parte del gruppo, o delle TIN la spinta all'apertura venga presa, secondo questa logica polarizzante dei pro e dei contro, solo come una rischiosa avventura ideologica, e non per quello che è, cioè una ponderata valutazione del rapporto rischi-benefici.

Dove la matrice gruppale tiene, il sistema di relazioni interne e il clima di gruppo hanno fatto sì che il processo trasformativo sia stato interiorizzato e sia diventato cultura, ritrovando un'umanità professionale dolente ma reattiva; dove invece la matrice si sfalda, abbiamo il ripiegamento in un tentativo di sopravvivenza individuale, di mera rivendicazione di ruolo, di autoisolamento, di rinuncia all'empatia che nelle situazioni più estreme diventano l'equivalente professionale della morte psichica.

\section{Il gruppo come antagonista}

della de-umanizzazione

Come mantenere la connessione con l'altro e l'empatia anche in condizioni estreme?

Marina Mojović che, dopo che le matrici gruppali e familiari sono state distrutte dagli orribili anni Novanta nella ex Jugoslavia, ha dato il via a gruppi di riflessione per reimmettere in gioco sogni e pensieri (reflectives citizens project), si chiede quali spiragli di luce possano riattivare connessioni nei rifugi psichici dal trauma [11].

Se lo è chiesto anche Anna Ornstein, sopravvissuta bambina ad Auschwitz, diventata poi psicanalista e già professoressa di psichiatria alla Harvard Medical School, tutt'ora impegnata, 93enne, nel tenere vivi parola e pensiero su questa esperienza. Nei lager, al conformismo necessario per sopravvivere all'esterno, faceva da contraltare il tentativo di preservare la propria identità e i propri valori: "Servivano flessibilità e creatività". "Ciò era possibile grazie alla creazione di piccoli gruppi sociali che funzionavano come famiglie, dove c'erano rivalità e ostilità ma anche molta lealtà e cura reciproca. I gruppi forniscono il setting dove gli affetti umani più comuni, l'ostilità, le gelosie, la lealtà, l'amore e la cura possono essere vissuti e manifestati. In questi gruppi c'erano liti, disaccordi e riconciliazioni, una dedizione fiera e molto sacrificio" [12]. Il tema della cura reciproca mi sembra centrale.

\section{Prendersi cura di chi cura}

"Prendersi cura di chi cura" è l'espressione più volte messa in campo nel corso degli ultimi decenni come risposta necessaria alle vicissitudini emotive proprie degli individui e dei gruppi che abitano le istituzioni di cura, non solo della salute mentale, dove strumenti come la supervisione d'équipe sono stati storicamente più frequentati. Se il gruppo di lavoro padroneggia le dinamiche descritte finora, ha molte più probabilità di mantenere la rotta sul proprio compito e a reggerne la complessità, anziché, imprigionato nelle proprie dinamiche interne, orientarsi verso scadenti scelte rassicuranti.

Fa parte di questa cura anche la diffusione di sportelli virtuali, singoli e di gruppo, che molti psicoterapeuti hanno messo a disposizione degli operatori in prima fila contro il Covid-19. Quanto ve ne sia bisogno lo dicono le notizie sul malessere anche molto pesante degli operatori o l'investimento di ospedali provvisti di risorse come il Mount Sinai di NY [13] che fin dall'inizio della pandemia hanno fornito e inserito nei protocolli il sostegno psicologico ai propri operatori, in cui è dichiarata la preoccupazione di mantenere integre nel breve e nel lungo periodo le risorse umane/professionali impiegate nella cura. Un'offerta peraltro predisposta da alcune strutture anche nel nostro Paese.

In occasione della pandemia potrebbe riprendere vigore l'attenzione alla predisposizione e mantenimento di dispositivi di cura destinati ai gruppi di lavoro, erroneamente considerati sempre più secondari, un lusso, 
nella deriva degli ultimi anni di continue revisioni e strette sulla spesa sanitaria.

\section{Uscire, tutti, dalla solitudine}

Ma i gruppi a cui appartiene il professionista della cura sono tanti, non solo quello di lavoro, che purtroppo non sempre va nella direzione che desideriamo. C'è il gruppo professionale, come le società scientifiche e le associazioni di appartenenza, quello tra pari come i genitori, dove possiamo trovare un conforto e anche il gruppo transitorio creato da occasioni di incontro legate a progetti comuni, convegni, ogni altra occasione più o meno stabile di connessione delle reti informali che tengono insieme le comunità di pratiche. Sono tutte situazioni, come possiamo tutti testimoniare, che ci permettono, anche dove siamo più isolati, di uscire dalla solitudine.

Scriversi e sentirsi tra colleghi, fare il punto sulle conquiste e sugli arretramenti è parte integrante di un processo gruppale resiliente e non regressivo. Sentire i genitori come appartenenti e ritenerli inclusi nella gruppalità del gruppo curante fa parte di questo. La frase "abbiamo bisogno voi" non è un espediente per fare empowerment: coinvolgere i genitori è la sincera ammissione che, se il genitore non viene messo in grado di fare la propria parte, non reagisce al proprio trauma e non trova il modo di costruire un'alleanza, allora non riuscirà a portare il suo insostituibile contributo e nell'operatore prevarrà, nella relazione con lui, l'esposizione al trauma secondario, amplificando la tentazione di rifugiarsi nel distanziamento e nella disconnessione.

Un messaggio come questo non può essere "formale", deve essere sentito, perché parafrasando lo psichiatra e psicoanalista Gaetano Benedetti, i pazienti possono perdonare gli errori della tecnica, non possono perdonare gli errori del cuore.

\section{leonardosperi@gmail.com}

1. bleger.org/la-relazione-gruppo-compito-in-psicoanalisi-operativa/

2. Fanelli $S$, Bellù $R$, Zangrandi $A$, et al. Managerial features and outcome in neonatal intensive care units: results from a cluster analysis. BMC Health Serv Res 2020 Oct 16;20(1):957
3. vivereonlus.com

4. www.glance-network.org/campaign/

5. Speri L, Bosio C. L'ossitocina intimidita. Atti del Convegno COIRAG "La violenza dei Legami. Riconoscerla, significarla, affrontarla”, Torino, 2014:322-35.

6. Hinshelwood RD. L'indifferenza. Un autismo quotidiano. funzionegamma.it/wp-content/uploads/1.Hinshelwood.pdf

7. Menzies I. Containing Anxiety in Institutions. Free Association Books, 1988.

8. expatalachians.com/prosperity-then-calamity-the-buffalo-creek-disaster

9. Kai T. Erikson. Everything in Its Path: Destruction of Community in the Buffalo Creek Flood. Simon \& Schuster, 1976.

10. Saraceno B. Salute Mentale nell'anno 2000: una questione urgente sia per i paesi industrializzati sia per quelli in via di sviluppo. medicinademocratica.org/article.php3?id_article $=22$

11. Mojović M. The Matrix Disrupted. Challenges and Changes. journals.sagepub.com/ doi/10.1177/0533316415613484

12. Ornstein, A. La funzione del gruppo in tempi di guerra e di terrore. Gruppi 2009;11(2):35-46.

13. mountsinai.org/files/MSHealth/Assets/ HS/About/Coronavirus/mh-guide.pdf; mountsinai.org/about/newsroom/2020/mount-sinai-announces-center-for-stress-resilienceand-personal-growth-pr

\section{E se un genitore chiede di escludere gli specializzandi dalla cura della propria figlia?}

L'articolo prende le mosse da una situazione reale 0 , quantomeno, realistica. Un pediatra in formazione chiede di potere eseguire personalmente un cateterismo vescicale in una bambina di 5 anni con pielonefrite. La manovra, abitualmente eseguita dal personale infermieristico, è giustificata dalla situazione clinica. Dopo avere spiegato al padre la procedura, lo specializzando si appresta a eseguirla assistito da un'infermiera. Purtroppo, il cateterismo richiede diversi tentativi, la bambina piange e appare alquanto infastidita durante tutta la manovra e il padre assiste al tutto con evidente preoccupazione. Anche l'infermiera vive la situazione con disagio e, terminata la procedura, consiglia al padre di discutere le sue perplessità con il pediatra in servizio ("Io strutturato", diremmo noi). II risultato del colloquio è che il padre chiede che gli specializzandi non eseguano più alcuna manovra sulla figlia.

Lo scenario è molto più complesso di quanto potrebbe apparire e viene affrontato attraverso il commento di un pediatra strutturato, di un direttore di scuola di specialità e di un eticista. I diritti e i doveri di ciascuno degli attori della narrazione (e anche di quelli che restano sullo sfondo) sono diversi e talora potenzialmente conflittuali. Ci sono, anzitutto, i diritti della bambina a ottenere le migliori cure possibili, poi quelli dello specializzando di procedere nel proprio percorso di apprendimento e, infine, quelli dei responsabili dell'istituzione che devono avere margine per decidere l'operatività in un ospedale accreditato per l'insegnamento. D'altra parte, i doveri sono altrettanto rilevanti: la corretta informazione ai genitori su chi fa cosa, il dovere di apprendere e di fare apprendere, quello di dare opportuna supervisione all'apprendimento, quello di valutare e garantire comunque il giusto rapporto costo-beneficio per il paziente. I commenti e le argomentazioni sono di grande interesse e devono essere letti con attenzione sia dagli specializzandi che da coloro che, a qualsiasi titolo, hanno la responsabilità di tutorare la loro formazione. Eccessive semplificazioni di questa problematica rischiano di farci sottovalutare insidie sulle quali potremmo non avere riflettuto a sufficienza.

1. Largent EA, Newman R, Gaw CE, Lantos JD. When a Family Seeks to Exclude Residents From Their Child's Care. Pediatrics 2020 Dec;146(6):e2020011007. 